\title{
Use of an Arterial Cannula in Intubated Children Secondary to Bronchiolitis is Associated with Multiple Blood Gas Sampling and Prolonged Ventilation
}

\author{
Shashikanth R Ambati*, Smiriti Sharma, James Schneider, Todd Sweberg and Sandeep Gangadharan, \\ Division of Pediatric Critical Care Medicine, Steven and Alexandra Cohen Children's Medical Center, USA
}

Submission: Jun 30, 2016; Published: July 29, 2016

*Corresponding author: Shashikanth Ambati, Division of Pediatric Critical Care Medicine, Bernard and Millie Duker Children's hospital, Albany Medical Center, 47 New Scotland Avenue, USA, Tel: 518-262-5127; Fax: 518262 2833; Email: shashiambati@gmail.com

\begin{abstract}
Background: Bronchiolitis is a common cause of respiratory failure in children. Respiratory failure, in the PICU, is often managed with the utilization of arterial cannulas and frequent arterial blood gas sampling. Despite the common use of arterial blood sampling, it is unclear if these tests improve outcomes.
\end{abstract}

Objective: To evaluate the frequency of blood draws for blood gas sampling and the duration of mechanical ventilation in children with respiratory failure with bronchiolitis in whom arterial cannel as were placed after initiation of mechanical ventilation.Setting: Children were recruited from a tertiary care children's hospital.

Design: Retrospective cohort study.

Setting: TPICU at a tertiary care children's hospital.

Patients: 109 children between 0 to 2 years with a diagnosis of bronchiolitis requiring mechanical ventilation.

Methods: A retrospective chart review was conducted on patients ages 0 to 2 years admitted to the PICU with a diagnosis of bronchiolitis who required invasive mechanical ventilation between May 2008 and June 2014. Data collected included demographics, ventilation duration, number and type of blood gases drawn, $\mathrm{PaO} 2 / \mathrm{FiO}_{2}$ and $\mathrm{SpO}_{2} / \mathrm{FiO}_{2}$ ratios at the time of intubation, and arterial cannula related complications.

Results: The mean duration of mechanical ventilation for patients with an arterial cannula was 7.9 (SEM \pm 0.57 ) days compared to 5.9 $(\mathrm{SEM} \pm 0.67)$ days in patients without an arterial cannula $(\mathrm{p}<0.04)$. The average number of blood gases drawn was 2.5 times higher in the arterial cannula group $(\mathrm{p}<0.0001)$.

Conclusion: The presence of arterial cannulas in children intubated for bronchiolitis is associated with increased duration of mechanical ventilation and increased frequency of blood gas sampling.

Keywords: Bronchiolitis; Arterial Cannulas; Arterial Blood Gases; Mechanical Ventilation; Intensive Care Unit

Abbreviations: ABG: Arterial Blood Gas; PICU: Pediatric Intensive Care Unit; MV: Mechanical Ventilation; $\mathrm{PaO}_{2}$ : Partial Pressure of oxygen in arterial blood; $\mathrm{FiO}_{2}$ : Fraction of inspired oxygen $\mathrm{SpO}_{2}$ : Oxygen saturation in blood

\section{Introduction}

Arterial cannulas are frequently used for invasive monitoring in critically ill patients. The most common indications to place an arterial cannel include the need for frequent blood sampling and/or continuous blood pressure monitoring. The placement of an arterial cannula in the management of bronchiolitis is primarily based on physician preference for blood sampling, as hemodynamic instability is not common.
Despite advances in non-invasive monitoring, including automated rapid cycling oscillometric blood pressure devices, pulse ox meters, transcutaneous oxygen monitoring, and end tidal carbon dioxide monitors, the utilization of invasive arterial catheters are still common in PICUs. Annual worldwide usage is reported as up to eight million in the United States and 2.5 million in Europe [1]. Arterial blood gases (ABGs) are one of the most common laboratory tests ordered in the intensive care unit. 
Makassar et al demonstrated that the presence of an arterial catheter was associated with the number of ABGs drawn per patient independent of all other measures of the patient's clinical status [2].

Arterial catheterization, while common in the critical care setting, is not without significant risks for morbidity. The most common complications associated with arterial puncture are pain, arterial injury and thrombosis with distal ischemia, infection, hemorrhage and aneurysm formation[3]. The incidence of arterial cannula-related infection in intensive care has been reported as 0.59 per 1000 catheter days with $0.34 \%$ developing catheterrelated blood stream infections [4]. Arterial cannula related blood stream infections are also associated with serious complications, including site pseudo aneurysms, septic thromboarteritis and arterial rupture. These infections carry a considerable risk of morbidity and mortality, as complications often require surgical intervention [5].

In addition to complications associated with the use of arterial cannulas, their use also may increase the financial burden to providing critical care with questionable added clinical value [6]. Capillary blood gas samples can accurately predict ABG values of $\mathrm{pH}, \mathrm{pCO} 2$ and $\mathrm{HCO} 3$ for patients with acute respiratory failure being treated with mechanical ventilation and do not require the placement of an invasive catheter [7].

Bronchiolitis is a common cause of respiratory failure in children, often necessitating admission to the pediatric intensive care unit (PICU) and invasive mechanical ventilation[8]. Respiratory failure secondary to bronchiolitis is often managed with the assistance of arterial cannulas and frequent $A B G$ sampling. Despite the common use of ABGs, it is unclear if frequent blood gas sampling improves outcomes. In our institution we have observed that there is a disparity in clinical practice among PICU attending physicians with regards to the use of arterial cannulas and ABGs to determine clinical care and the pace of weaning patients off mechanical ventiliation in children intubated for respiratory failure secondary to bronchiolitis. In this retrospective cohort study at a single center tertiary care PICU, we evaluate the relative frequency of blood gas sampling and the associated length of mechanical ventilation in children with respiratory failure from bronchiolitis who are initiated on mechanical ventilation.

\section{Material and Methods}

In this study, we conducted a retrospective review of patients with an admission diagnosis of respiratory failure secondary to bronchiolitis admitted to Cohen Children's Medical Center of NY (CCMC) PICU. Inclusion criteria included age 0 to 2 years, diagnosis of bronchiolitis requiring mechanical ventilation, and admission between May, 2008 and June, 2014. Patients with a primary or secondary diagnosis of pneumonia, required inotropes or vasopressors and/or extracorporeal membrane oxygenation were excluded. Data collected included age, gender, history of prematurity, duration of mechanical ventilation, presence of chronic comorbidities, presence of an arterial cannulas, number and type of blood gases drawn, and any arterial cannula related complications (e.g. thrombosis). Patients were divided into one of two cohorts depending on whether or not they had an arterial cannula placed after admission for respiratory failure and initiation of mechanical ventilation.

To compare disease severity of the two groups, heart rate (HR), respiratory rate (RR), and $\mathrm{SpO} 2 / \mathrm{FiO} 2$ ratio calculated at the time of intubation were recorded. In the A-line group, HR, RR and $\mathrm{PaO} 2 / \mathrm{FiO} 2$ ratios were also calculated at the time of arterial catheter placement. For the cohort of patients without arterial catheter or arterial blood gas sampling, the $\mathrm{PaO} 2$ was estimated from the oxygen hemoglobin dissociation curve using the last recorded oxygen saturation prior to intubation as outlined by Aboab et al. [9] and Brockway et al. [10] Categorical variables were analyzed using the Fisher exact test and continuous variables were analyzed using a t-test (Minitab 14). A p $<0.05$ was considered statistically significant. The study was approved by the North Shore Long Island Jewish Institutional Review Board (IRB).

\section{Results}

109 patients met inclusion criteria and 76 (70\%) had an arterial catheter. In the arterial catheter cohort, mean age was 7.6 months, $58 \%$ were male, $43 \%$ had a history of prematurity, and $46 \%$ had comorbidities. Table 1 shows that for the group of patients without an arterial cannula, the mean age was 6 months $(p=0.24), 61 \%$ were male $(p=0.84), 42 \%$ had a history of were prematurity $(p=1.0)$, and $64 \%$ had comorbidities $(p=0.1)$. The chronic comorbidities in both cohorts included chronic lung disease, cerebral palsy, congenital heart disease (Ventricular Septal defect and coarctation), Down's syndrome and DiGeorge syndrome. No arterial catheter related complications were reported.

Table1: Baseline characteristics of children with bronchiolitis on mechanical ventilation.

\begin{tabular}{|c|c|c|c|}
\hline Demographic factor & $\begin{array}{c}\text { A-cannula } \\
\text { Present }\end{array}$ & $\begin{array}{c}\text { No } \\
\text { A-cannula }\end{array}$ & p- value \\
\hline Average Age (months) & 7.6 & 6 & 0.24 \\
\hline Total no of Males & 44 & 20 & 0.84 \\
\hline $\begin{array}{c}\text { No of children with } \\
\text { Prematurity }\end{array}$ & 33 & 14 & 1.0 \\
\hline $\begin{array}{c}\text { No of children with Co } \\
\text { morbidities }\end{array}$ & 35 & 21 & 0.1 \\
\hline Sp02/FiO $_{2}$ ratio & 210 & 215 & 0.76 \\
\hline Pao2/FiO ${ }_{2}$ ratio & 189 & 186 & 0.83 \\
\hline $\begin{array}{c}\text { Respiratory rate(RR)/ } \\
\text { min }\end{array}$ & 47 & 51 & 0.19 \\
\hline Heart rate(HR)/min & 125 & 129 & 0.40 \\
\hline
\end{tabular}

The last documented HR, RR, PaO2/FiO2 and Sp02/FiO2 ratios prior to intubation were recorded for each patient included in the study analysis. The two cohorts were compared by a student t-test to determine whether the severity of illness between the 
two cohorts were similar as shown in (Table 1). In the cohort of patients with an arterial cannula, the cannula was placed within 2 hours of the patient intubation. The p-value for each demographic and disease characteristic was not significant. Table 2 shows that the difference in mean duration of mechanical ventilation and the total and average number of blood gases drawn in both the groups were statistically significant.

Table 2: Duration of mechanical ventilation and total number of blood gases in children with bronchiolitis on mechanical ventilation.

\begin{tabular}{|c|c|c|c|}
\hline & A-line present & No A-line & p-value \\
\hline Mean duration of MV in days & 7.9 & 5.9 & $<0.04$ \\
\hline Total number of blood gases & 1953 & 341 & $<0.0001$ \\
\hline $\begin{array}{c}\text { Average number of blood } \\
\text { gases }\end{array}$ & 26 & 10 & $<0.0001$ \\
\hline
\end{tabular}

Abbreviations: A cannula: Arterial cannula; No: number; MV: Mechanical Ventilation; $\mathrm{SpO}_{2}$ : Oxygen saturation in blood; $\mathrm{PaO}_{2}$ : Partial

Pressure of oxygen in arterial blood; $\mathrm{FiO}_{2}$ : Fraction of inspired oxygen

\section{Discussion}

To date, this is the first study to examine the duration of mechanical ventilation in children intubated for bronchiolitis managed with and without an arterial cannula for frequent blood gas sampling. Our analysis suggests children intubated for respiratory failure secondary to bronchiolitis may be managed successfully without the insertion of an arterial cannula and frequent sampling of ABGs. The mean duration of mechanical ventilation in the arterial cannula group in our study was equivalent to previously reported length of intubation for a child with bronchiolitis ( $8 \pm 3$ days), [11] indicating that our arterial cannula group was typical in disease severity for patients admitted for respiratory failure secondary to bronchiolitis. While the duration of invasive mechanical ventilation was significantly less in the cohort of children who did not have an arterial cannula, they had similar HR, RR, $\mathrm{PaO} 2 / \mathrm{FiO} 2$ and $\mathrm{SaO} 2 / \mathrm{FiO} 2$ ratios at the time of intubation.

Multiple studies have demonstrated that venous or capillary blood gases are sufficient for approximating arterial pH, PCO2 and HCO3 for patients requiring mechanical ventilation secondary to respiratory failure in an intensive care unit, negating the need for invasive arterial catheterization in these patients [12-16]. Additionally, non-invasive monitoring methods such as pulseoximetry and end-tidal capnography utilized in conjunction with infrequent capillary or venous sampling can be an alternative strategy to frequent $A B G$ sampling. Due to advent of these technologies, indwelling arterial cannulas are less commonly utilized [17].

Despite the costs and potential risks of arterial cannulas and the presence of non-invasive monitoring methods, the use of arterial cannulas for monitoring of patients with respiratory failure remains common [18]. Furthermore, it remains unclear if the presence of arterial cannulas and frequent ABG sampling improves patient outcome [19]. In a review of a multicenter clinical trial on the use of prone positioning for pediatric acute lung injury, Khemani et al. [20] demonstrated that of over 11,000 intubated and mechanically ventilated children, at least 15\% did not have an arterial cannula. Interestingly, those without an arterial cannula received a similar level of mechanical ventilator support compared to children with an arterial cannula suggesting that the presence of arterial blood sampling was not associated with substantial difference in mechanical ventilation management strategies [20].

In our single center retrospective cohort study we found that the presence of an arterial cannula was associated with a longer duration of mechanical ventilation than the cohort managed without an arterial cannula. There are many possible reasons that may account for this finding. One possibility may be that weaning mechanical ventilation utilizing blood gas sampling adds delay due to the time required for the laboratory results to be completed and reported. Another possibility might be that details from the arterial blood gas such as an exact $\mathrm{PaO} 2$ or $\mathrm{pH}$ and $\mathrm{PaCO} 2$ may delay the pace of weaning if blood gas results rather than assessment of the work of breathing is given precedence. These results suggest that the presence of arterial cannulas and frequent ABG sampling may not have benefit in the care of patients with bronchiolitis and respiratory failure and is, in our study, associated with a longer duration of mechanical ventilation compared to a cohort with similar indices of respiratory illness but without arterial cannulas.

Lewis et al. [6] demonstrated that the presence of an arterial cannula is associated with increased blood draws in patients in the ICU. In our study, we found that the average number of blood gases drawn was 2.5 times higher in the cohort with an arterial cannula compared with the cohort without an arterial cannula $(\mathrm{p}<0.0001)$. Our findings suggest that in addition to a lack of an association of benefit in children with arterial cannulas with respect to duration of mechanical ventilation, the catheters contribute to a greater number of blood draws for blood gas sampling.

While our results were significant and warrant further prospective studies evaluating the benefits of ABG sampling in children mechanically ventilated for bronchiolitis, we recognize our analysis has limitations. Our study was a retrospective cohort design of a single center and there may be factors such as individual clinician variability in mechanical ventilation weaning practice. However, our multi-disciplinary ICU, much like others, takes a team based approach to the management of our patients and multiple attendings, critical care fellows, residents and nurses play a collaborative role in the care of each patient. Additionally, it is possible that our two study cohorts of bronchiolitic respiratory failure had different epidemiologies of viral triggers. While this is certainly possible given that only a small number of bronchiolitis associated viruses are routinely screened for, we do not believe that such a bias is likely given that there has never been a policy or practice in our ICU requiring arterial cannula placement based on associated virus or any other epidemiologic facet of bronchiolitis. While we believe that our choice of how to compare severity 
of lung disease in the two groups is consistent with accepted practice in the literature, we did extrapolate $\mathrm{PaO} 2$ from an $\mathrm{SpO} 2$ for the group managed without arterial cannulas. This practice of extrapolation has precedence in that a recent large multicenter clinical trial of sedation management for respiratory failure in children utilized the same methodology in reporting their results [21]. Additionally, the recent publication of consensus definitional criteria for pediatric acute respiratory distress syndrome has advocated the use of SpO2/FiO2 rather than $\mathrm{PaO} 2$ in the absence of invasive monitoring [22]. Additionally, while the timing of the vital sign measurements in our analysis were within 2 hours of intubation for all patients, it is possible some patients' clinical status changed appreciably in that interval and there level of illness was not accurately represented by our data.

\section{Conclusion}

In our retrospective cohort study in children managed with and without arterial cannulas and ABG sampling, the presence of arterial cannulas was associated with a longer duration of mechanical ventilation and increased frequency of blood gas sampling. There are substantial risks that are associated with the arterial cannulas that may outweigh the putative benefits. Significant consideration should be given to the need for placement of arterial cannulas in the management of children with bronchiolitis requiring invasive mechanical ventilation. Further studies evaluating the risk versus benefit of invasive monitoring, such as arterial line cannulas, in common critical illness is warranted.

\section{References}

1. Gowardman JR, Lipman J, Rickard CM (2010) Assessment of peripheral arterial catheters as a source of sepsis in the critically ill: a narrative review. The Journal of hospital infection 75(1):12-18.

2. Muakkassa FF, Rutledge R, Fakhry SM, Meyer AA, Sheldon GF (1990) ABGs and arterial lines: the relationship to unnecessarily drawn arterial blood gas samples. The Journal of trauma 30(9): 1087-1093.

3. Roberts JR, Hedges JR (2009) Clinical procedures in emergency medicine. Elsevier Health Sciences 308-322.

4. Lorente L, Santacreu R, Martin MM, Jimenez A, Mora ML (2006) Arteria catheter-related infection of 2,949 catheters. Critical care 10(3): R83.

5. O’Horo JC, Maki DG, Krupp AE, Safdar N (2014) Arterial catheters as a source of bloodstream infection: a systematic review and metaanalysis. Critical care medicine 42(6): 1334-1339.

6. Low LL, Harrington GR, Stoltzfus DP (1995) The effect of arterial lines on blood-drawing practices and costs in intensive care units. Chest 108(1): 216-219.

7. Chu YC, Chen CZ, Lee CH, Chen CW, Chang HY, et al. (2003) Prediction of arterial blood gas values from venous blood gas values in patients with acute respiratory failure receiving mechanical ventilation. Journal of the Formosan Medical Association 102(8): 539-543.

8. Mansbach JM, Piedra PA, Stevenson MD, et al. (2012) Prospective multicenter study of children with bronchiolitis requiring mechanical ventilation. Pediatrics 130(3): e492-500.

9. Aboab J, Louis B, Jonson B, Brochard L (2006) Relation between $\mathrm{PaO2} / \mathrm{FIO} 2$ ratio and FIO2: a mathematical description. Intensive care medicine 32(10): 1494-1497.

10. Brockway J, Hay WW (1998) Prediction of arterial partial pressure of oxygen with pulse oxygen saturation measurements. The Journal of pediatric 133(1): 63-66.

11. Carroll CL, Sala KA, Zucker AR, Schramm CM (2010) Factors Associated With ICU Length of Stay in Children With Bronchiolitis and Respiratory Failure. CHEST Journal 138(4).

12. Bilan N, Behbahan AG, Khosroshahi AJ (2008) Validity of venous blood gas analysis for diagnosis of acid-base imbalance in children admitted to pediatric intensive care unit. World journal of pediatrics WJP 4(2): 114-117.

13. Harrison AM, Lynch JM, Dean JM, Witte MK (1997) Comparison of simultaneously obtained arterial and capillary blood gases in pediatric intensive care unit patients. Critical care medicine 25(11): 1904-1908.

14. Kirubakaran C, Gnananayagam JE, Sundaravalli EK (2003) Comparison of blood gas values in arterial and venous blood. Indian journal of pediatrics $70(10)$ : 781-785.

15. Razi E, Nasiri O, Akbari H, Razi A (2012) Correlation of arterial blood gas measurements with venous blood gas values in mechanically ventilated patients. Tanaffos 11(4): 30-35.

16. Zavorsky GS, Cao J, Mayo NE, Gabbay R, Murias JM (2007) Arterial versus capillary blood gases: a meta-analysis. Respiratory physiology \& neurobiology 155(3): 268-279.

17. Merlani P, Garnerin P, Diby M, Ferring M, Ricou B (2001) Quality improvement report: Linking guideline to regular feedback to increase appropriate requests for clinical tests: blood gas analysis in intensive care. BMJ (Clinical research edn.) 323(7313): 620-624.

18. Gershengorn HB, Garland A, Kramer A, Scales DC, Rubenfeld G, et al. (2014) Variation of arterial and central venous catheter use in United States intensive care units. Anesthesiology 120(3): 650-664.

19. Garland A (2014) Arterial lines in the ICU: a call for rigorous controlled trials. Chest 146(5): 1155-1158.

20. Khemani RG, Markovitz BP, Curley MA (2009) Characteristics of children intubated and mechanically ventilated in 16 PICUs. Chest 136(3): 765-771.

21. Curley MA, Wyp ij D, Watson RS (2015) Protocolized sedation vs usual care in pediatric patients mechanically ventilated for acute respiratory failure: a randomized clinical trial. JAMA 313(4): 379-389.

22. Pediatric acute respiratory distress syndrome: consensus recommendations from the Pediatric Acute Lung Injury Consensus Conference (2015). Pediatric critical care medicine: a journal of the Society of Critical Care Medicine and the World Federation of Pediatric Intensive and Critical Care Societies 16(5): 428-439. 